\title{
UNDECIDABILITY OF MODULE HOMOMORPHISMS
}

\author{
K. H. KIM AND F. W. ROUSH
}

(Communicated by Bhama Srinivasan)

\begin{abstract}
For finitely additively generated torsion free modules and rings, we show existence of module monomorphisms is decidable but of epimorphisms is undecidable.
\end{abstract}

What structural problems in algebra are decidable? Grunewald and Segal [2] have recently shown a wide variety of isomorphism problems are decidable by a method of finding fundamental domains. (See $[\mathbf{5}, \mathbf{6}]$.) Matijasevic's negative solution to Hilbert's tenth problem has many undecidability consequences for algebra. Many problems in combinatorial group theory are undecidable.

A particularly simple class of problems in algebra because of their linearity, are module problems. Here we show the monomorphism problem is decidable for finitely additively generated torsion free modules, and the epimorphism problem is undecidable. Remeslennikov proved for nilpotent groups the epimorphism problem is undecidable.

Here our rings and modules are finitely additively generated unless otherwise specified. They are specified by giving a basis and products of basis elements. Additively they are torsion free. We consider (1) the monomorphism problem: given a ring $\mathfrak{R}$, modules $\mathfrak{M}, \mathfrak{N}$ can we find a monomorphism $h: \mathfrak{M} \rightarrow \mathfrak{N}$, and (2) the epimorphism problem: can we find an epimorphism $h: \mathfrak{M} \rightarrow \mathfrak{N}$ ? By finding we mean algorithmic solvability as with Hilbert's problem.

Solvability of the isomorphism problem is contained in [2].

THEOREM 1. The monomorphism problem is decidable.

PROOF. Tensor $\mathfrak{M}, \mathfrak{N}$ with the real numbers $\mathbf{R}$. Then a monomorphism is an algebraic problem over the real numbers which can be decided because the first order theory of the real numbers is decidable. We may take the determinant of a suitable minor of a matrix to be 1 .

Approximate this linear mapping by a (linear) module homomorphism $\mathfrak{M} \otimes \mathfrak{Q} \rightarrow$ $\mathfrak{N} \otimes \mathfrak{Q}$. A sufficiently close approximation has the minor with nonzero determinant. Now multiply it by a number to make it integral.

THEOREM 2. The problem of, given a linear space (by additive generators) $\mathfrak{L}$ of matrices of nonnegative determinant over $\mathbf{Z}$, of deciding whether $M \in \mathfrak{L}$ with $\operatorname{det} M=1$ is undecidable. We may assume $\mathfrak{L}$ is a summand of $M_{n}(\mathbf{Z})$ additively.

Received by the editors August 20, 1986 and, in revised form, September 15, 1987.

1980 Mathematics Subject Classification (1985 Revision). Primary 16A64.

Key words and phrases. Algorithmic decidability, module epimorphisms.

Partially supported by NSF DMS 8521533. 
ProOF. The class of Diophantine equations $f\left(x_{1}, \ldots, x_{n}\right)=1$, where $f$ is a homogeneous polynomial, $f \geq 0$ is not decidable. Given any Diophantine equation $g$ over $\mathbf{Z}$ write it as $(g+1)^{2}=1$. Replace all variables by sums and differences of squares so that only even powers of all variables occur. Add an even positive power of a variable $t$ on each term on the left to make it homogeneous and divisible by $t$. The result is equivalent to the original $g=0$ since if we have a solution of $f=1$ and $t \mid 1$ so $t= \pm 1, t^{2}=1$. Conversely, $t=1$ solves the new given a solution of $g$.

Now it suffices, given a homogeneous polynomial $f$, to construct a matrix whose entries are linear combinations of $x_{i}$ and whose determinant is $f t^{2 m}$ for some $m$. Start with a $1 \times 1$ matrix $[f]$. We will expand this matrix so as to make the entries linear.

To simplify sums into their monomials, we change

$$
\left[\begin{array}{cc}
a+b & c \\
d & e
\end{array}\right] \text { to }\left[\begin{array}{ccc}
t & 0 & 0 \\
a & a+b & c \\
0 & d & e
\end{array}\right] \text { to }\left[\begin{array}{ccc}
t & -t & 0 \\
a & b & c \\
0 & d & e
\end{array}\right]
$$

This introduces no new nonmonomial terms if $a$ is a monomial, and multiplies the determinant by $t$ and elements in the same row have equal degree.

To simplify a product we change

$$
\left[\begin{array}{cc}
a b & c \\
d & e
\end{array}\right] \text { to }\left[\begin{array}{ccc}
t & a & 0 \\
0 & a b & c \\
0 & d & e
\end{array}\right] \text { to }\left[\begin{array}{ccc}
t & a & 0 \\
-b t & 0 & c \\
0 & d & e
\end{array}\right]
$$

where $a$ is a single variable $x_{i}$.

This process converts all monomials to those of the form $\pm x_{i} t^{s}$, and $n_{0} t^{s}, n_{0} \in \mathbf{Z}$ and preserves homogeneity of rows. Determinants are multiplied by $t$.

When all monomials are converted, factor out a power of $t$ from each row to make the matrix linear. Now take a direct sum with copies of $[t]$ to make the power of $t$ as desired, and to have at least one entry $t$. Since all $\pm x_{i}, t$ appear as entries, we have a summand of $M_{n}(\mathbf{Z})$.

Grunewald and Segal [2] proved a result related to the following

PROPOSITION 3. Let $\mathfrak{R}=M_{n}(\mathbf{Z})$ act linearly on a sum of $s$ copies of the standard representation in each summand. The class of problems given a vector $v$ and subspace $W$ does there exist a unit of $\mathfrak{R}$ mapping $v$ into $W$ is undecidable for some $n, t$.

ProOF. We may take Diophantine equations of fixed degree in a fixed number of variables. This will give matrices of a given size in Theorem 2 .

Take a space $\mathfrak{L}$ of matrices as in Theorem 2 . Let $\mathfrak{R}$ abstractly be $M_{n}(\mathbf{Z})$ acting by a sum of copies of the standard representation $\rho_{i}$.

Represent $\mathfrak{L}$ as a solution set of equations $\sum a_{i j} m_{i j}=0$ for integer $a_{i j}$. Write each as $\sum v_{j} M w_{j}=0$, where $w_{j}=(0,0, \ldots, 1, \ldots, 0)$ with a 1 in place of $j$ only, and $\left(v_{j}\right)_{i}=a_{i j}$.

Now let $v$ be the vector made up of all vectors $v_{j}$ in the different factors. Let $W$ be the subspace such that in the set $W_{k}$ of components corresponding to a given equation,

$$
W_{k}=\left\{\left(u_{1}, \ldots, u_{r}\right): \sum u_{j} w_{j}=0\right\}
$$


Then in this set of components $v M \subset W$ is equivalent to $\sum a_{i j} m_{i j}=0$, i.e. $M \in \mathfrak{L}$.

An element $M$ of $\mathfrak{L}$ is a unit if and only if $\operatorname{det} M= \pm 1$ if and only if $\operatorname{det} M=1$, since $\operatorname{det} M \geq 0$.

THEOREM 4. There exists a ring $\mathfrak{R}$, additively spanned by a finite basis $\left\{1, t_{i}\right\}$, where $t_{i} t_{j}=0$ for all $t_{i}, t_{j}$ such that, for finitely additively generated modules over $\mathfrak{R}$, the epimorphism problem is undecidable.

PROOF. We show a dual monomorphism problem (for additively split monomorphisms) is undecidable. Let the module of Proposition 3 be $V=\sum_{i=1}^{s} V_{i}$, acted on by $M_{n}(\mathbf{Z})$ where $V_{i}$ is rank $n$. Let $V_{0}$ be isomorphic to $V_{i}$, let $\mathbf{Z}_{1}$ be a copy of $\mathbf{Z}$ with generator $v_{0}, W_{1}$ isomorphic to $W$.

As modules take $V \oplus V_{0} \oplus \mathbf{Z}_{1}, V \oplus V_{0} \oplus W_{1}$. Let generators $t_{i}$ of the ring act by being zero on $\mathbf{Z}_{1}, W_{1}, V$, and send $V_{0}$ isomorphically to $V_{i}$. Then

$$
t_{i} t_{j}=t_{j} t_{i}=t_{i}^{2}=0
$$

Let generators $t_{0}$ be 0 on $V \oplus V_{0}$, send $v_{0}$ to $v$ and $W_{1}$ isomorphically to $W$. Let the ring be spanned by 1 and $t_{i}$.

If a unit $\mu$ of $M_{n}(\mathbf{Z})$ sends $v$ into $W$, define a module split monomorphism which is $\mu$ on $V_{0} \oplus V$ and from $\mathbf{Z}_{1}$ to $W_{1}$ be such that $v_{0}$ goes to $t_{0}^{-1}(\mu v)$.

Conversely, let a module split monomorphism exist. Then $V=\bigcap \operatorname{ker}\left\{t_{i}\right\}$ is sent to itself by some linear map. The subspace $V \oplus V_{0}=\operatorname{ker}\left\{t_{0}\right\}$ is also preserved. The map on $V$ must agree with a map $\mu$ on

$$
\frac{V_{0} \oplus V}{V}
$$

to itself for commutativity with $t_{i}$ to hold, $i>1$. So on $V$ we have action of a unit $\mu$ in $M_{n}(\mathbf{Z})$. And this must send image $t_{0}$ to itself so $v$ goes into $W$.

Existence of an epimorphism on the dual $\operatorname{Hom}(\mathfrak{K}, \mathbf{Z})$ of a torsion free finitely generated module is equivalent to existence of an additively split monomorphism into $\mathfrak{K}$. It suffices to check this for $\mathbf{Z}$ modules, i.e. abelian groups. An epimorphism of free abelian groups is additively split.

We remark that Theorem 4 is of interest for problems like Boyle, Marcus, Trow [1, Theorem 3.2].

ACKNOWLEDGMENT. The authors would like to thank the referee for very constructive suggestions on the first draft of this paper.

\section{REFERENCES}

1. M. Boyle, B. Marcus, and P. Trow, Resolving maps and the dimension group for shifts of finite type, preprint, Univ. of Maryland, College Park, Maryland, 1987.

2. F. Grunewald and D. Segal, Some general algorithms. I. Arithmetic groups, Ann. of Math. 112 (1980), 531-583. 
3. K. H. Kim and F. W. Roush, Some results on decidability of shift equivalence, J. Combin. Inform. System Sci. 4 (1979), 123-146.

4. __ Decidability of shift equivalence (Proc. Workshop on Dynamical Systems, Univ. of Maryland, College Park, Md., 1986), Springer-Verlag, (to appear).

5. R. A. Sarkisian, Galois cohomology on some questions in the theory of algorithms, Math. USSR Sb. 39 (1981), 519-545.

6. __ A problem of equality in Galois cohomology, Algebra i Logika 19 (1980), 707-725. (Rus$\operatorname{sian})$

Mathematics Research Group, Alabama State University, Montgomery, AlABAMA 36195 